\title{
CHEMICAL GRADIENTS IN THE ORION MOLECULAR CLOUD
}

\author{
H. UNGERECHTS, E. A. BERGIN, J. CARPENTER, P. F. GOLDSMITH, \\ W. M. IRVINE, A. LOVELL, D. MCGONAGLE, F. P. SCHLOERB, AND R. L. SNELL \\ Five College Radio Astronomy Observatory \\ University of Massachusetts, Amherst, MA 01003, USA
}

ABSTRACT. We are mapping 29 rotational transitions of 21 chemical and isotopic molecular species in the central Orion molecular ridge with Nyquist sampling using the new 15-element focal plane array receiver QUARRY on the FCRAO $14 \mathrm{~m}$ telescope. Our goal is to obtain complete, unbiased data sets for a study of the interrelated physics and chemistry in GMC cores.

It has become commonplace in astronomy to use the strength of molecular rotational transitions measured at millimeter wavelengths to deduce physical parameters of interstellar clouds, such as temperature, density, and structure. In almost all cases the assumption is made that the chemical composition of the source being studied is uniform. On the other hand, models of the chemistry of molecular clouds predict that the composition will depend on a variety of variables including evolutionary history and radiation flux. Clearly, if chemical gradients are present, they may seriously compromise efforts to deduce physical parameters from the strength of molecular emission lines.

We are investigating this problem by mapping the central Orion molecular cloud in a variety of molecular transitions. Apart from the standard tracers of molecular gas at low and high densities, $\mathrm{CO}$ and its isotopic variants and CS, we chose to map important ions and radicals, the isomeric pair $\mathrm{HCN}$ and $\mathrm{HNC}, \mathrm{CH}_{3} \mathrm{OH}$, and $\mathrm{C}_{3} \mathrm{H}_{2}$. Physical conditions in the clouds can be estimated from the $\mathrm{CO}$ lines (temperature, total column density), the $\mathrm{HC}_{3} \mathrm{~N}$ line ratio (density), and the $\mathrm{CH}_{3} \mathrm{CCH}$ line ratio (temperature). The maps have 360 pixels on a $0.41^{\prime}$ grid and cover an area of 4.5 by 12 arc minutes. They include the Orion-KL region in the center, the northern region where many ions and radicals peak, and the molecular ridge near the ionization front in the South.

These maps confirm qualitatively that there are significant differences among the distribution of various species. Most molecules, like CO and CS, show the Orion ridge extending north-south with a maximum toward Orion-KL as well as the crescent-shaped ridge at the ionization front in the South. By comparison, $\mathrm{N}_{2} \mathrm{H}^{+}$and the radicals, CN and $\mathrm{C}_{2} \mathrm{H}$, are rather weak toward Ori-KL and stronger to the Northeast. The intensity of $\mathrm{HCN}$ is strongly enhanced near $\mathrm{KL}$ but its isomer, $\mathrm{HNC}$, does not have such a dominant peak. The maps of $\mathrm{SO}$ and $\mathrm{CH}_{3} \mathrm{OH}$ show two major maxima, the stronger toward $\mathrm{KL}$ and the weaker about $1.5^{\prime}$ south; at much lower intensity, the emission from both species is extended along the ridge.

We have started similar surveys in the Cepheus A and M17SW molecular clouds, and we plan to extend this work to a larger sample of molecular clouds spanning a wide range of physical and chemical conditions.

\section{Contour Maps}

Line intensity integrated over velocity for selected lines. Position offsets are relative to $\alpha_{50}=5^{\mathrm{h}} 32^{\mathrm{m}} 46.8^{\mathrm{s}}, \delta_{50}=-5^{\circ} 24^{\prime} 28^{\prime \prime}$ (Orion-KL). The lowest contour and the separation between contours in units of $\mathrm{K} \mathrm{km} \mathrm{s}^{-1}$ are 80 and 40 for $\mathrm{CO} J=1 \rightarrow 0$; both are 4 for ${ }^{13} \mathrm{CO}, 0.5$ for $\mathrm{C}^{18} \mathrm{O}, 4$ for $\mathrm{CS} J=2 \rightarrow 1,0.5$ for $\mathrm{C}^{34} \mathrm{~S}, 0.2$ for $\mathrm{CH}_{3} \mathrm{CCH} J=6 \rightarrow 5 \mathrm{~K}=1$, 4 for $\mathrm{HCO}^{+} J=1 \rightarrow 0,0.2$ for $\mathrm{H}^{13} \mathrm{CO}^{+}, 1$ for $\mathrm{N}_{2} \mathrm{H}^{+} J=1 \rightarrow 0 F_{1}=2 \rightarrow 1,1.5$ for $\mathrm{CN}$ $N=1 \rightarrow 0 J=3 / 2 \rightarrow 1 / 2 F=5 / 2 \rightarrow 3 / 2$ and $F=3 / 2 \rightarrow 1 / 2,0.5$ for $\mathrm{C}_{2} \mathrm{H} N=1 \rightarrow 0$ $J=1 / 2 \rightarrow 1 / 2 F=1 \rightarrow 1,10$ for $\mathrm{HCN} J=1 \rightarrow 0,1$ for $\mathrm{H}^{13} \mathrm{CN}, 2$ for $\mathrm{HNC} J=1 \rightarrow 0$, 1 for $\mathrm{HC}_{3} \mathrm{~N} J=10 \rightarrow 9,1.5$ for $\mathrm{SO} J_{K}=3_{2} \rightarrow 2_{1}$, and 0.5 for $\mathrm{CH}_{3} \mathrm{OH} J_{K}=2_{0} \rightarrow 1_{0} \mathrm{~A}^{+}$. For $\mathrm{HCN}$ and $\mathrm{H}^{13} \mathrm{CN}$ the line intensity was integrated over all hyperfine components. 

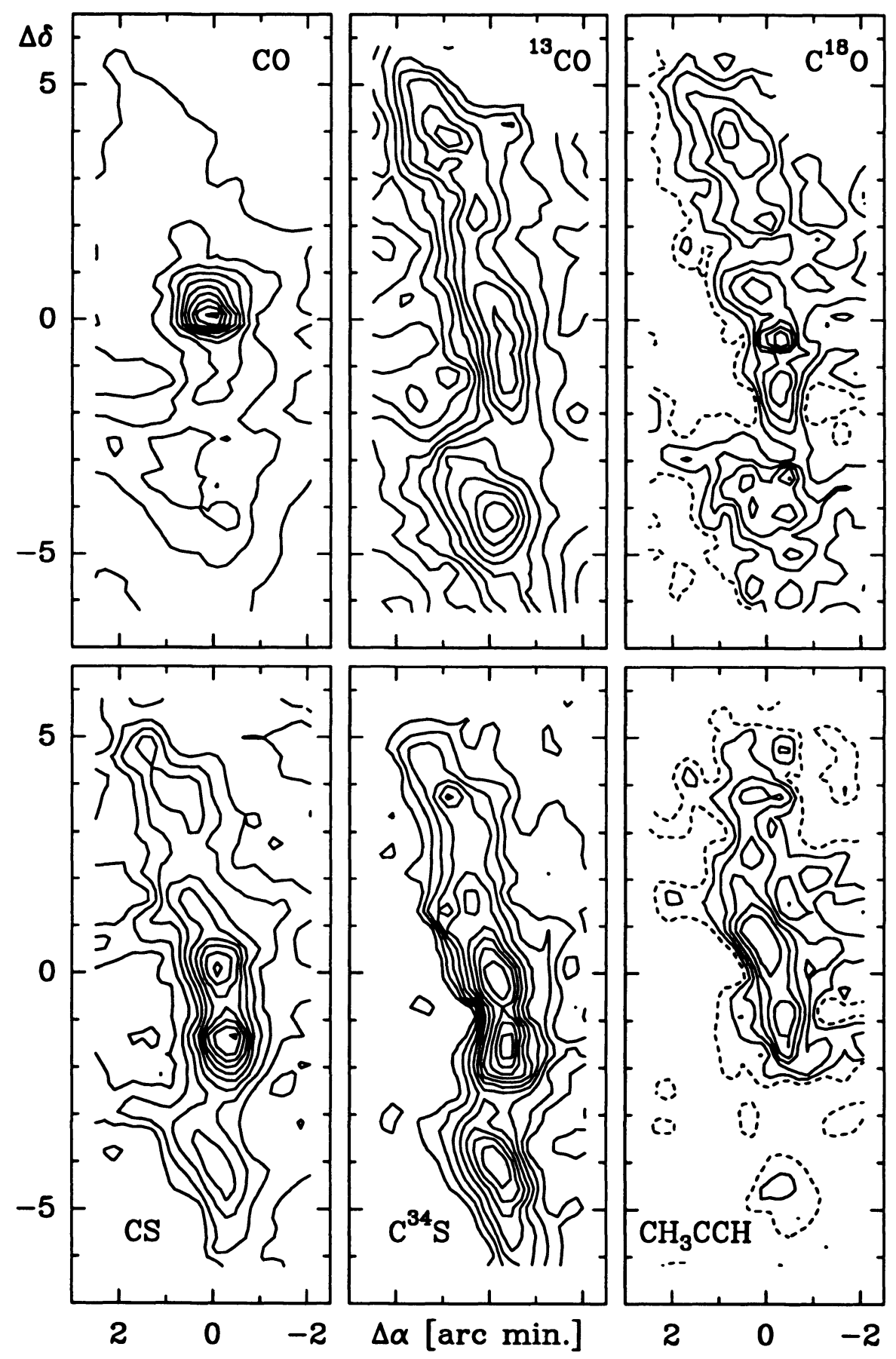

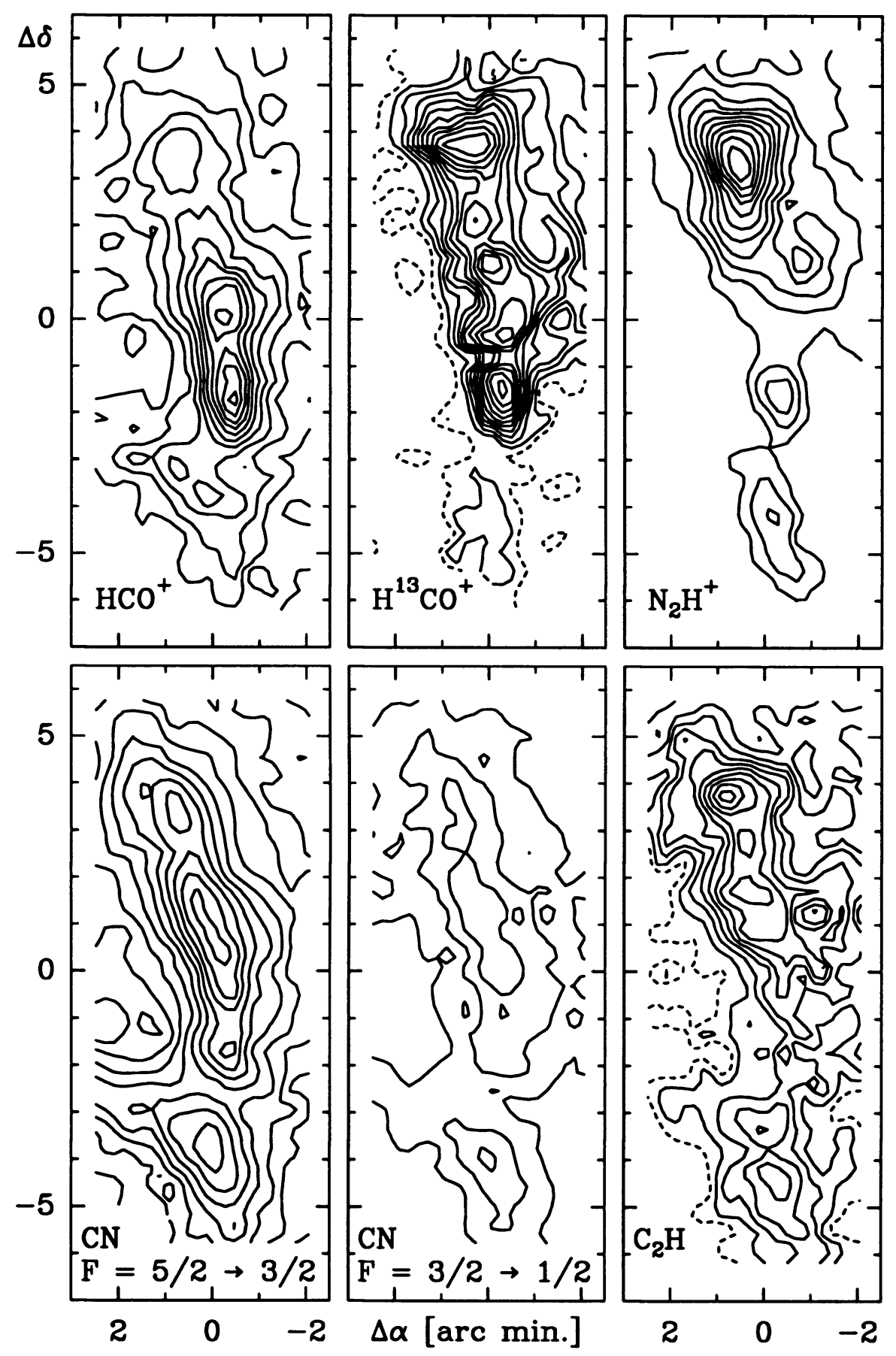

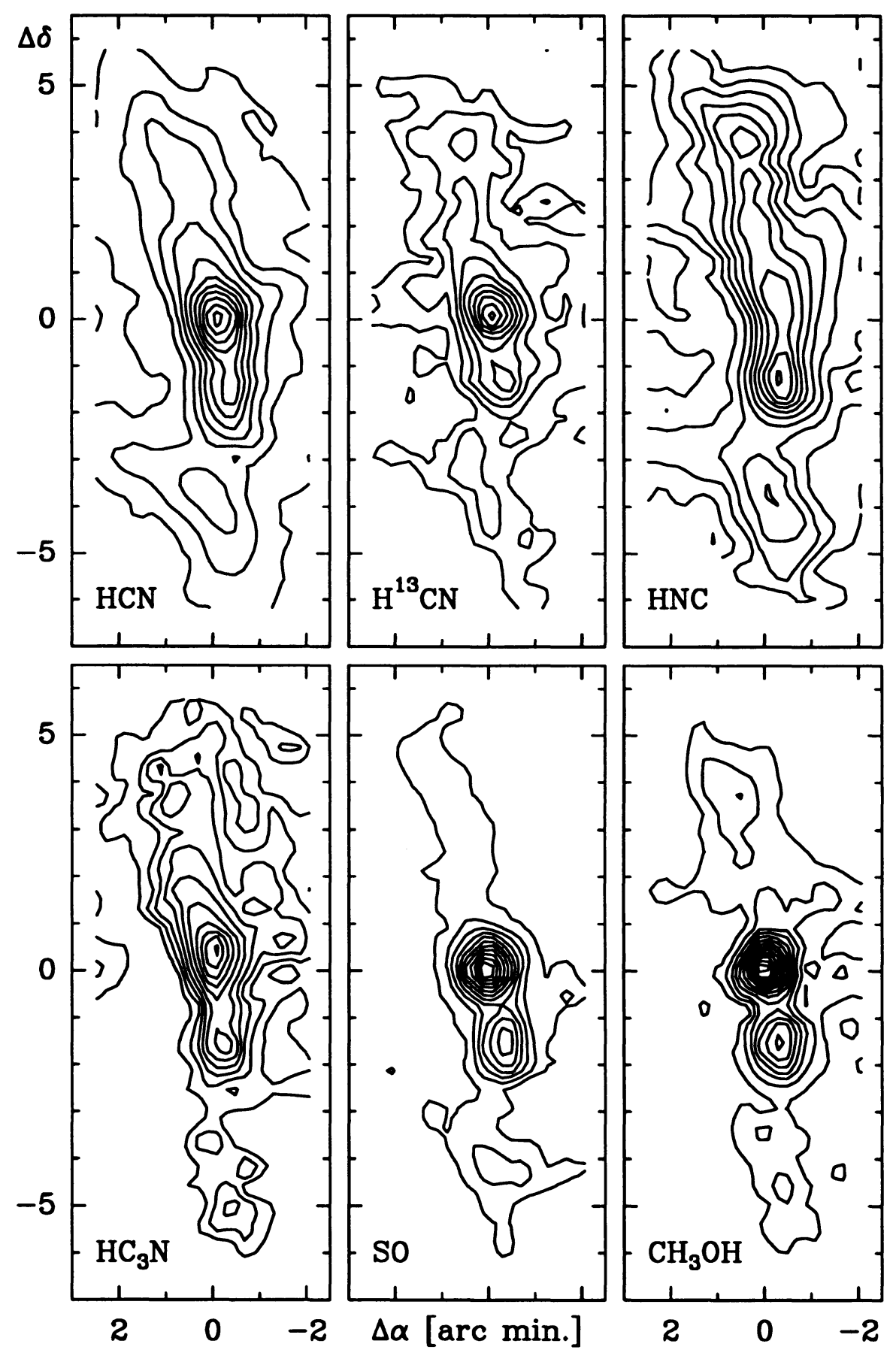